\title{
Téoros
}

Revue de recherche en tourisme

\section{La demande touristique dans les Laurentides : bilan de santé}

\section{Jean Stafford}

Volume 15, numéro 1, printemps 1996

Les Laurentides, quelles Laurentides?

URI : https://id.erudit.org/iderudit/1075047ar

DOI : https://doi.org/10.7202/1075047ar

Aller au sommaire du numéro

Éditeur(s)

Université du Québec à Montréal

ISSN

0712-8657 (imprimé)

1923-2705 (numérique)

Découvrir la revue

Citer cet article

Stafford, J. (1996). La demande touristique dans les Laurentides : bilan de santé. Téoros, 15(1), 14-17. https://doi.org/10.7202/1075047ar d'utilisation que vous pouvez consulter en ligne.

https://apropos.erudit.org/fr/usagers/politique-dutilisation/ 


\title{
LA DEMANDE TOURISTIQUE \\ dans les LAURENTIDES: BILAN de SANTÉ
}

\author{
Jean Stafford
}

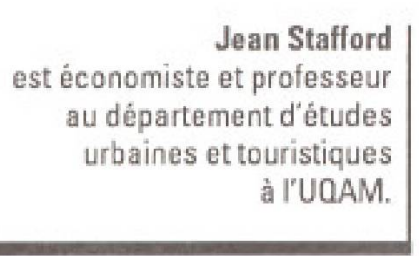

Jean Stafford

La réglon touristique des Laurentides dispose d'atouts Importants: des centaines de lacs et de rlvlères. des montagnes d'hauteur moyenne et un bon réseau routler. A cela s'ajoute une grande diversité dans l'hébergement et plusleurs centres majeurs de villéglature. Notre bllan dolt dépasser blen sûr la simple description du cadre naturel; l'offre touristlque étant blen connue, et peu susceptsble de varler avec le temps, nous allons surtout scruter la demande tourlstique.

La mellleure façon de cerner cette demande est d'analyser dans le détall l'état et l'évolution (à l'aide de séries chronologlques) des chambres occupées dans cette réglon. C'est le seul indicateur vralment touristique utilisable au plan statıstıq̣ue. Nous allons décomposer cette demande en éléments: mouvements tendanciels, salsonnlers, cycliques et Irrégullers.

\section{Examen de certaines statistiques de base}

Dans le tableau 1, nous avons certaines statistlques de base concernant la demande tourlstique. Dans un premler temps. nous pouvons constater l'écart Important entre la moyenne des chambres disponlbles et la moyenne des chambres occupées: écart qul se tradult dans un taux d'occupation moyen très près des limites de la survile financlère. Nous pouvons remarquer que les chambres disponlbles varlent très peu avec le temps (coefficient de variation de $6,1 \%$ et taux de crolssance annuel de $1,1 \%$ ). Les chambres occupées ont un coefflclent de varlation assez élevé $(25,2 \%)$ et un taux de crolssance annuel négatif $(-1,5 \%)$. II y a donc au départ un problème d'ajustement de l'offre (pléthorlque) et de la demande: ce qui inaugure mal pour l'aventr.

\section{Analyse de quelques fonctions touristiques}

Le tableau 2 présente certaines fonctions touristiques dans la région des Laurentides (pour 1000/habitants) ainsi que pour l'ensemble du Québec. La réglon étudlée compte 3 ctabilissements de restauration pour mille habitants alors que la moyenne québécolse est de 2,9. Pour les chambres disponlbles, II y a peu de différence entre les Laurentides et le Ouebec (16,8 et 16,3 pour mille habitants). Le nombre de chambres occupées pour mille habitants est pratiquement cinq fols plus falble pour les Laurentides comparées à l'ensemble du Québec $(1,9$ a 9,8$)$. Pour les indlcateurs "voyages-personne- et *dépenses touristiques*, la performance de la réglon touristique des Laurentides est supérieure à celle de l'ensemble du Québec; Il faut noter que la différence est plus falble en ce quil concerne les dépenses touristiques.

\section{L'origine des clientèles touristiques}

Dans le tableau 3, nous avons le nombre des voyageurs et les recettes tourlstiques dans la réglon des Laurentides selon l'orlgine des voyageurs. Pour le nombre des voyages effectués dans cette réglon, ce sont les Québécols qui sont les plus nombreux avec $85.7 \%$ de tous les voyages; les Québécols et les autres canadlens forment près de $95 \%$ de la clientèle touristique. Les voyageurs américains comptent pour $3.7 \%$ de l'ensemble et les résidents des autres pays pour $1,6 \%$. Ces données Indiquent que la réglon des Laurentides n'est pas une destination Internationale et qu'elle ne dessert que la grande région de Montréal.

Au plan des recettes touristiques, les Québécols représentent $63,2 \%$ des recettes dans cette réglon; ce pourcentage est beaucoup plus falble que celul des voyageurs. Les vlsiteurs des États-Unls qui comptent pour 3.7 des voyageurs fournissent $18,8 \%$ de l'ensemble des recettes et les autres pays $5.1 \%$. Lés Québécols dépensent donc. en moyenne, 69 \$dans la réglon des Laurentides alors que les Américalns dépensent 474 \$ et les autres visiteurs étrangers 307 \$ par voyage!

\section{Autopsie de la demande touristique}

La tendance des chambres occupées est fable (quas! nulle) et négative. Par contre, les mouvements salsonnlers sont extrểmement importants. Le tableau 4 montre les coefflclents salsonnlers des chambres occupées (par mols) pour la réglon des Laurentides et pour l'ensemble du Québec (cette comparalson apparait auss dans le graphique 1). Il y a un fort contraste pour less coefficients de décembre, Janvier, février et mars; par la suite (avril, mal et juin) les coefficients sont plus falbles que ceux de l'ensemble du Québec. Les mols de juillet et août sont à peu près semblables. Il y a ensulte une balsse plus forte du mols de septembre au mols de novembre. SI lon compare les deux séries de coefficients, nous aurons:

\begin{tabular}{|ll|}
\hline \multicolumn{2}{|l|}{ Mois où le coefficient est supérieur à 100: } \\
\hline Région des Laurentides & Ensemble du Québec \\
\hline Janvier & \\
Février & \\
Mars & Juin \\
Juillet & Juillet \\
Août & Aoút \\
& Septembre \\
& Octobre \\
\hline
\end{tabular}




\section{TABLEAU 1}

Certaines statistiques de base concernant l'hébergement dans la région touristique des Laurentides*

\begin{tabular}{|lcccc|}
\hline Indicateurs & Moyenne & Écart-type & $\begin{array}{c}\text { Coefficient } \\
\text { de variation en \% }\end{array}$ & $\begin{array}{c}\text { Taux de croissance } \\
\text { annuel en \% }\end{array}$ \\
\hline Chambres disponibles & 5,825 & 355 & 6,1 & 1,1 \\
Chambres occupées & 2,043 & 515 & 25,2 & $-1,5$ \\
Taux d'occupation & 35 & 8 & 22,8 & $-2,6$ \\
\hline
\end{tabular}

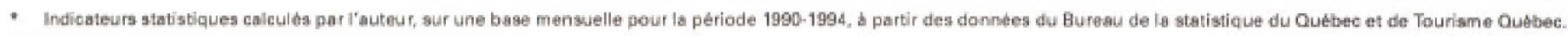

\section{TABLEAU 2}

Certaines fonctions touristiques dans la région touristique des Laurentides (pour 1000/habitants)

\begin{tabular}{|lcc|}
\hline Fonctions & $\begin{array}{c}\text { Région touristique } \\
\text { des Laurentides } \\
\text { (pour 1000/habitants) }\end{array}$ & $\begin{array}{c}\text { Moyenne pour l'ensemble } \\
\text { du Québec } \\
\text { (pour 1000/habitants) }\end{array}$ \\
\hline Nombre d'établissements & 3,0 & \\
de restauration* & 16,8 & 2,9 \\
Chambres disponibles* & 1,9 & 16,3 \\
Chambres occupées* & 6,274 & 9,8 \\
Voyages-personne** & $588,052 \$$ & 4,140 \\
Dépenses touristiques** & & $562,600 \$$ \\
\hline
\end{tabular}

Sources: Statiatique Conada, Gu resu de la statistique du Quebec et Tou riame Quebec.

- Calcules par l'auteuf a partir des données de 1994.

* Calculés par l'auteur à partir dea donnetes de 1992.

\section{TABLEAU 3}

Le nombre des voyageurs et les recettes touristiques dans la région touristique des Laurentides en 1992 selon l'origine des voyageurs

\begin{tabular}{|lcccc|}
\hline Origine des voyageurs & \multicolumn{2}{c|}{ Nombre de voyages } & \multicolumn{2}{c|}{ Recettes touristiques } \\
\hline & Nombre (000) & $\%$ & Recettes (000 \$) & $\%$ \\
\hline Québec & 2050 & 85,7 & 141,600 & 63,2 \\
Canada (hors Québec) & 215 & 9,0 & 28900 & 12,9 \\
Etats-Unis & 89 & 3,7 & 42200 & 18,8 \\
Autres pays & 37 & 1,6 & 11400 & 5,1 \\
Total & 2391 & 100 & 224100 & 100 \\
\hline
\end{tabular}

Donnetes: Statistique Canada

\section{TABLEAU 4}

Coefficients saisonniers* des chambres occupées pour la région des Laurentides et l'ensemble du Québec, par mois

\begin{tabular}{|lllllllllllll|}
\hline Région/Mois & Jan. & Fév. & Mars & Avril & Mai & Juin & Juillet & Août & Sept. & Oct. & Nov. & Déc. \\
\hline Laurentides & 107 & 124 & 108 & 67 & 78 & 91 & 138 & 135 & 104 & 87 & 66 & 95 \\
\hline $\begin{array}{l}\text { Ensemble } \\
\text { du Québec }\end{array}$ & 71 & 89 & 83 & 80 & 99 & 117 & 138 & 145 & 125 & 103 & 81 & 68 \\
\hline
\end{tabular}

- Calculea par la methode X-11 du Bureau of the Census (U.S.A.). 
Lă région des Laurentides va donc se distinguer par la salson hivernale (par rapport àl'ensemble du Québec). La réusslte des Laurentides dépendra donc de l'or blanc et des deux princlpaux mols de l'étés. Le phenomene salsonnler semble donc incontournable!

Dans le tableau 5, nous avons les écarts-lypes de la distribution des coefficlents salsonnlers, cycliques et Irrégullers. L'influence prépondérante du mouvement salsonnler apparaît clalrement (avec un écart-type de 24.9 \%) heureusement, le mouvement salsonnler peut assez facllement. à court terme, être mesuré et modélisé. Le mouvement cyclique (pour la pérlode considéróe 1990-1994) est assez falble $(5 \%)$; ce qui indlque l'Influence limitẻe de l'économie globale sur l'industrie réglonale du tourisme. Le mouvement irrégulter semble encore plus fable que le mouvement cyclique (avec un écart-type de $1.9 \%$ ); la sérle n'est et ne sera donc pas perturbée par des mouvements Irréguliers intempestufs! Nous sommes donc face à une tendance trèss falble, un mouvement salsonnler très important et des mouvements cycliques et irréguliers négligeables!

\section{Une prévision figée}

La faible tendance et la très forte saisonnalité rendent pratiquement inutile, sinon sur une forme monotone, toute étude prévislonnelle. Le modèle que nous avons conçu, et qui apparaît dans le tableau 6 , a pour seul avantage d'indlquer l'apport de chacun des mols à l'ensemble de la série des chambres occupées dans la réglon des Laurentides.

Nous pouvons remarquer:

1. que la constante (1998 chambres occupees) est assez pres de la moyenne obtenue:

2- que chacune des périodes nouvelles (la variable temps dans l'équation) n'apporte rien à l'ensemble:

3- le mols de fanvier amene 201 chambres occupées (à la constante):

4- le mols de révrler apporte 580 chambres occupées (II est le plus important de l'hiver);

5- le mols de mars est plus ćlevé que janvier (260 chambres occupées) mals plus falble que févrler:

6- le mols d'avril est négatuf et slgnifle une perte de 627 chambres occupées, c'est le mols le plus *Coûteux. en terme de perte:

7- le mols de mal correspond à une perte de 358 chambres occupeses:

8- le mols de juin avec une perte (assez fable malgré tout) de 110 chambres occupees;

9- le mois de fuillet, le plus florissant avec un gain de 870 chambres occupées;

10- le mols d'aoút avec un gain de 811 chambres occupées:

11 - le mois de septembre avec un ajout de 258 chambres occupées;

12- le mols d'octobre correspond à une balsse (faible) de 165 chambres occupees:

13- Ie mols de novembre nous apporte 580 chambres occupées de molns:

14- enlin "le mois de décembre correspond à la constante de 1998 chambres occupées.

\section{Un avenir brouillé}

La crolssance très falble de l'offre des chambres dispontbles et la demande stagnante des chambres occupees rendent assez lacile les prévisions à faire. Il est plus difficlle d'expllquer ce niveau zéro du développement tourlstique: on ne peut que dégager les éléments qui font la force et la faiblesse de celte région touristique.

La princlpale force de la réglon des Laurentides réside dans son capital Nature-Espace qui fait appel à des aspirations compatibles avec la soctété post-moderne émergente: Illusion d'un monde écologique où l'Individu peut poursulvre son salut personnel et clôturé! Un autre ếlément positif est la grande diversité des paysages et des sites, des équipements de pleln-alr et des possiblitués de *lolsirs naturels.. Une autre force apparaît dans la capacité de conserver et de développer des activités dans les pérlodes creuses d'hiver (volr le tableau 3).

Une falblesse vient du viellissement de loffre (velle mals non aanciennew) qui falt qu'il est plus difficle d'en falre un produit mouveau* (elle n'a pas la patine anclenne des vielles choses:). Une autre source de falblesse (qul blzarrement devrait etre une force), c'est la proximité de Montréal et de ses innombrables banlleues: la région des Laurentides est devenue un immense parc de lolsir et non une destination tourlstique digne de ce nom.

Les autres causes de la falblesse de cette réglon seront démographlques et économlques. Le viellissement accéléré de la population québécolse va réduire les besolns pour les activités de plein-air et les sports plus aventureux. Au plan economique, l'offre touristique est trop axée sur des ressources naturelles souvent Interchangeables, peu spéclfiques et soumises aux avalars salsonnlers.

Le grand déli des prochalnes années pour la réglon tourlsLlque des Laurentides est de conciller sa vocation de pare de loisir avec celle de devenir une véritable destination touristique (à la fois nationale et internationale). Cette destination touristlque devralt s'établir en jouant àla fols sur l'Histoire (celle des plonnlers de la colonisation) et celle d'une Nature accessible et accueillante.

NOTLS MÉTHODOLOGIOULS

1- Le coeficient de variation (Lableau 1) est une estimation des fluctuations: plus ce coefficient se rapproche de 0 of et moins il y a de fluctuation dans oette population. pour la variable tudice.

2. Le taux de crolssance annuel (tablean 1) est calcule par une movenne geomitrique et pour les ann6es 1990-1994.

3- Le coefmcient saisonnier (tableau 4) se rósume al lapport d'un mois donné par ramport a la movenne annuelle; aingi dang le tableau 4 , le coefficient saigonf ier du mois de janvier, pour la région des Lau mentides, est de 107 ; ce gqu weut dire que le mois de janvicr génère 7 qu de plus de chambres ocoupeses que la inopenne annuelle. Pour lensemble du Oudbeo, le coefficient du mois de janvier est de 71 oe qui nous indique que a mols a 29 to de moins de chambres occapes que la moyenne de Fensemble du puebec. Ces coclicients soat calcolls a partir dune methode developped au -U.S. Burea of the Cenaws; catte methode porte le nom de aCensus II X11. A ce sujet, voir. $G$. Mélard. Méthodes de prévision di court terme, Editions Ellipses-Uatwersited de Bruxelles, IBruxelles, 1990, chapitre 5. II raut nouer que les coelticients du tableau 5 sont aussi calculás à partir de cetwe méthode.

4- Lígquation du modôle prévisionel qui apparait dans le tableau 6 est une répression multiple qui identufe les mois a des variables muetes; at ce moment. cos variables prennent, hl tour de rôle, dans le modête, la valeur 0 ou 1 . 


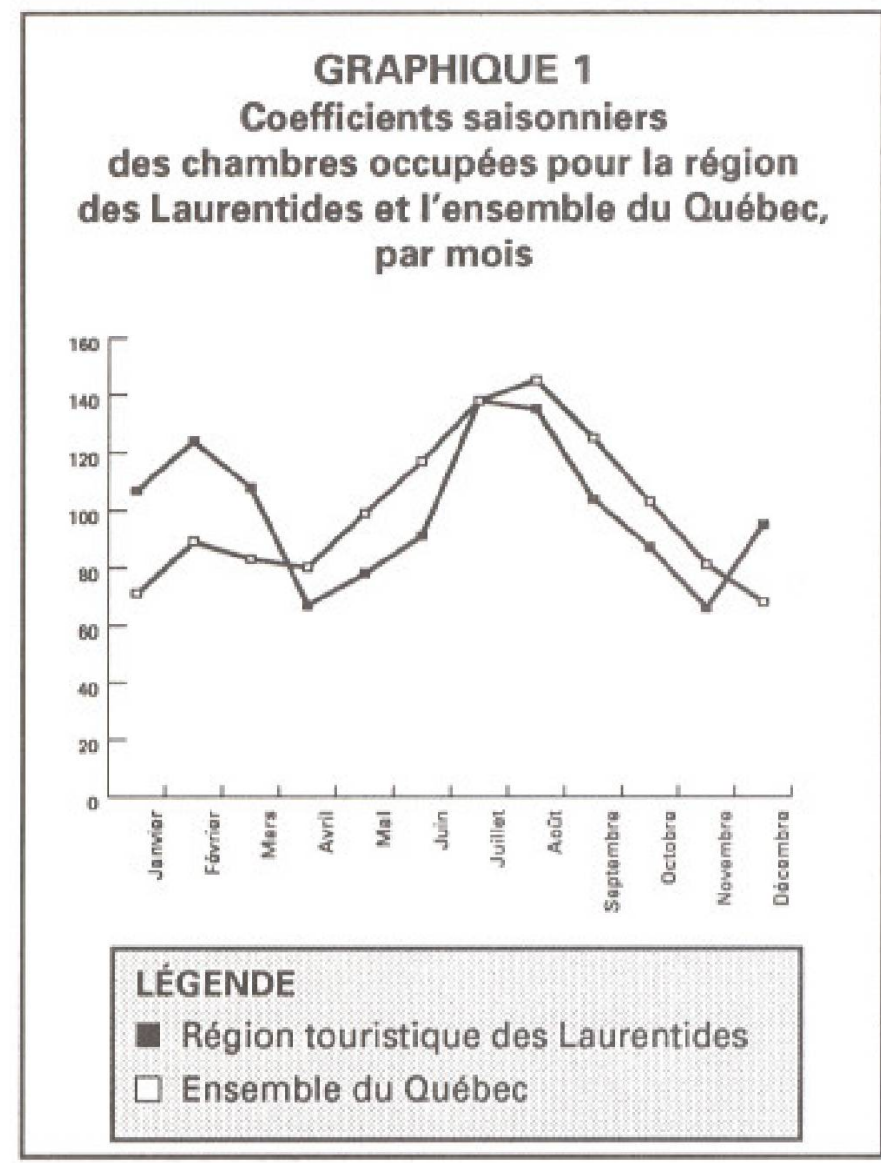

\section{TABLEAU 5}

Les écarts-types de la distribution des coefficients* saisonniers, cycliques et irréguliers des chambres occupées pour la région touristique des Laurentides pour la période 1990-1994

\begin{tabular}{|lc|}
\hline Coefficients & Écarts-types en $\%$ \\
\hline Saisonniers & 24,9 \\
Cycliques & 5,0 \\
Irréguliers & 1,9 \\
\hline
\end{tabular}

- Calcules par la mathode X-11 du Buresu of the Cenaus IU.S.A.I.

\section{TABLEAU 6}

Modèle prévisionnel des chambres occupées dans la région touristique des Laurentides; chambres occupées, par mois, pour la période 1990-1994

\begin{tabular}{|c|c|c|c|c|c|}
\hline $\mathrm{CO}=$ & $\begin{array}{l}1998 \\
(114)\end{array}$ & $=$ & $\begin{array}{l}2 \text { (temps) } \\
\text { (1) }\end{array}$ & + & $\begin{array}{l}201 \text { (jan.) } \\
(139)\end{array}$ \\
\hline+ & $\begin{array}{l}580 \text { (fév.) } \\
(139)\end{array}$ & + & $\begin{array}{l}260 \text { (mars) } \\
(138)\end{array}$ & - & $\begin{array}{l}627 \text { (avril) } \\
(138)\end{array}$ \\
\hline - & $\begin{array}{l}358 \text { (mai) } \\
(138)\end{array}$ & - & $\begin{array}{l}110 \text { (juin) } \\
(138)\end{array}$ & + & $\begin{array}{l}870 \text { (juil.) } \\
(138)\end{array}$ \\
\hline $\begin{array}{l}+ \\
-\end{array}$ & $\begin{array}{l}811 \text { (aouีt) } \\
(138) \\
580 \text { (nov) } \\
(138)\end{array}$ & + & $\begin{array}{l}258 \text { (sept.) } \\
(138)\end{array}$ & - & $\begin{array}{l}165 \text { (oct.) } \\
(138)\end{array}$ \\
\hline $\begin{array}{lr} & \mathrm{CO}= \\
\mathrm{R} 2 \quad= & \\
\mathrm{F} & = \\
\mathrm{DW} & = \\
\text { Test de } & \mathrm{St} \\
\mathrm{n} & =\end{array}$ & $\begin{array}{l}\text { Chambres } \\
0,86 \\
24,01 \\
1,614 \\
\text { aramétres: } \\
60\end{array}$ & es & 0,10 & & \\
\hline
\end{tabular}

- Calculda a partir du projiciel S,As. 